\title{
Photoacoustic properties of anterior ocular tissues
}

Kelsey P. Kubelick

Eric J. Snider

C. Ross Ethier

Stanislav Emelianov 


\title{
Photoacoustic properties of anterior ocular tissues
}

\author{
Kelsey P. Kubelick, ${ }^{a}$ Eric J. Snider, ${ }^{a}$ C. Ross Ethier, ${ }^{a, \star}$ and Stanislav Emelianov ${ }^{a, b, \star}$ \\ ${ }^{a}$ Georgia Institute of Technology and Emory University School of Medicine, Wallace H. Coulter Department of Biomedical Engineering, \\ Atlanta, Georgia, United States \\ ${ }^{\mathrm{b}}$ Georgia Institute of Technology, School of Electrical and Computer Engineering, Atlanta, Georgia, United States
}

\begin{abstract}
Clinical imaging techniques for the anterior segment of the eye provide excellent anatomical information, but molecular imaging techniques are lacking. Molecular photoacoustic imaging is one option to address this need, but implementation requires use of contrast agents to distinguish molecular targets from background photoacoustic signals. Contrast agents are typically selected based on a priori knowledge of photoacoustic properties of tissues. However, photoacoustic properties of anterior ocular tissues have not been studied yet. Herein, anterior segment anatomy and corresponding photoacoustic signals were analyzed in brown and blue porcine eyes ex vivo. Measured photoacoustic spectra were compared to known optical absorption spectra of endogenous chromophores. In general, experimentally measured photoacoustic spectra matched expectations based on absorption spectra of endogenous chromophores reported in the literature, and similar photoacoustic spectra were observed in blue and brown porcine eyes. However, unique light-tissue interactions at the iris modified photoacoustic signals from melanin. Finally, we demonstrated how the measured PA spectra established herein can be used for one application of molecular PA imaging, detecting photoacoustically labeled stem cells in the anterior segment for glaucoma treatment. () The Authors. Published by SPIE under a Creative Commons Attribution 4.0 Unported License. Distribution or reproduction of this work in whole or in part requires full attribution of the original publication, including its DOI. [DOI: 10 .1117/1.JBO.24.5.056004]
\end{abstract}

Keywords: ophthalmology; anterior segment; ultrasound imaging; photoacoustic imaging; spectroscopic imaging; stem cell tracking. Paper 180517RR received Aug. 27, 2018; accepted for publication Apr. 19, 2019; published online May 21, 2019.

\section{Introduction}

The most-investigated approaches for noninvasive assessment of internal ocular structures have been ultrasound (US) and optical imaging techniques. ${ }^{1-9}$ Clinical use of ultrasound biomicroscopy and optical coherence tomography (OCT) are well established in ophthalmology. ${ }^{1,2,10}$ OCT provides excellent anatomical information by measuring changes in backscattered light. ${ }^{6}$ Advances in OCT now allow real-time imaging, widefield imaging, higher resolution, and assessment of other tissue optical properties, such as birefringence. ${ }^{10-14}$ Overall OCT research has drastically improved the quality of anatomical imaging in ophthalmology. However, molecular imaging techniques are lacking. Accordingly, development of molecular contrast OCT seeks to address this need ${ }^{15-17}$ by manipulating backscattering properties to distinguish molecular targets. ${ }^{17}$ One approach is to introduce exogenous contrast agents that increase backscattering. However, high background signals from surrounding tissue still make it difficult to distinguish molecular targets. As a result, researchers have invented other clever solutions, including magnetomotive OCT and US, spectroscopic-OCT, pump-probe OCT, and photothermal OCT. ${ }^{17-19}$

Significant effort has recently been dedicated to development of photothermal OCT for the retina. ${ }^{20-23}$ In photothermal OCT, an optical absorber is irradiated with light. Heat deposition causes thermoelastic expansion of the surrounding tissue and ultimately changes the optical path length measured by OCT. ${ }^{20}$ Thus, photothermal OCT obtains molecular information by indirectly measuring changes in backscattering resulting from an absorption event. A more straightforward approach may

*Address all correspondence to C. Ross Ethier, E-mail: ross.ethier@bme .gatech.edu; Stanislav Emelianov, E-mail: stas@gatech.edu be to use an imaging modality that directly measures optical absorption, such as photoacoustic (PA) imaging.

PA imaging is similar to photothermal OCT in that irradiation of an optical absorber results in heat deposition and thermoelastic expansion of surrounding tissues. However, conditions used in PA imaging produce transient acoustic waves detected by a traditional ultrasound transducer. ${ }^{24-26}$ Thus, PA imaging combines advantages of optical and acoustic modalities to allow high resolution, high contrast, real-time, molecular imaging. A variety of endogenous and exogenous optical absorbers produce PA signals. ${ }^{27}$ These optical absorbers serve as PA contrast agents and are detected using multiwavelength spectroscopic PA imaging. Because PA contrast is based on absorption, molecular information complements anatomical information provided by scattering-based modalities such as OCT or ultrasound. Thus, ultrasound and photoacoustic (US/PA) imaging systems are frequently combined, which is advantageous due to the established use of ultrasound in clinical ophthalmology., 5,,28 Similarly, a multimodal PA/OCT system has also been demonstrated for molecular imaging at the posterior segment to assess the retina. $^{29,30}$

Our work focuses on extending molecular PA imaging to the anterior segment of the eye, i.e., tissues anterior to the vitreous humor. Anterior segment imaging techniques, such as OCT, slitlamp microscopy, and ultrasound biomicroscopy, primarily provide anatomical information. ${ }^{10}$ There is a need for molecular imaging techniques that could aid diagnosis, guide therapies, and improve understanding of anterior ocular diseases, including glaucoma, uveitis, and iridial pathologies ${ }^{17}$ Implementation of molecular PA imaging requires endogenous or exogenous contrast agents that must be carefully selected based on $a$ priori knowledge of PA properties of anterior segment tissues to ensure the contrast agent or interventional devices can be distinguished from background PA signals. ${ }^{31-33}$ Although PA imaging has 
been demonstrated at a select few wavelengths in the anterior segment, ${ }^{34-36}$ exploration of PA properties over a broad range of wavelengths is required to inform contrast agent selection for molecular PA imaging.

Thus, the goal of the work presented here was to establish PA spectral properties for the anterior segment over a range of wavelengths corresponding to the ideal tissue optical window. ${ }^{27}$ In some cases, PA spectra in biological tissues directly correspond to optical absorption spectra of endogenous chromophores, i.e., melanin, oxygenated, and deoxygenated hemoglobin. Alternatively, PA spectra can be modified by light-tissue interactions. ${ }^{37}$ We hypothesized that the latter scenario may occur in the anterior segment due to the role of anterior tissues to control light propagation. Differences in eye color, resulting from unique combinations of scattering and melanin absorption at the iris, may also modify PA spectra. ${ }^{38,39}$ For these reasons, we systematically measured the PA spectral properties of anterior segment structures in blue and brown porcine eyes, which are a relevant model of human anatomy and pigmentation. We also demonstrated how these PA spectra can aid development of molecular PA imaging strategies in the anterior eye, specifically by informing contrast agent selection to detect stem cells for glaucoma treatment. ${ }^{40-42}$

\section{Materials and Methods}

\subsection{Eye Preparation}

All experiments used enucleated porcine eyes (Holifield Farms, Covington, Georgia). Blue and brown porcine eyes were studied to represent the two extremes in the range of eye color from light to dark. Blue or brown eye color was determined by visual inspection, and no other colors were observed in samples from the slaughterhouse. Fresh and fixed eye globes were imaged. Eye globes were fixed by submersion in $10 \%$ neutral buffered formalin overnight before imaging, then maintained in phosphate-buffered saline (PBS) prior to use. In total, 10 brown (three fresh and seven fixed) and 6 blue porcine eyes (two fresh and four fixed) were imaged. Minimal differences between PA spectra of fresh and fixed samples were observed (data not shown).

\subsection{Imaging Protocol}

A Vevo 2100/LAZR (VisualSonics, Inc., Toronto, Canada) imaging system, incorporating US/PA imaging modes, was used for all imaging experiments. US/PA images were acquired using a 256-element transducer array operating at $20-\mathrm{MHz}$ (LZ250) or 40-MHz (LZ550) center frequencies. The laser source was a Q-switched Nd:YAG laser-pumped optical parametric oscillator system (pulse repetition frequency $=20 \mathrm{~Hz}$, 7 -ns pulse duration). PA images were acquired within the 680- to $970-\mathrm{nm}$ wavelength range at a frame rate of $5 \mathrm{~Hz}$. Variations in laser pulse energy were corrected at the time of data acquisition using built in features of the Vevo 2100/LAZR.

In the first set of brown eye data, US/PA images were acquired at $1 \mathrm{deg}$ increments as the eye was rotated $360 \mathrm{deg}$. Data were collected using both the 20- and 40-MHz transducers, and the wavelength was varied from 680 to $970 \mathrm{~nm}$ in increments of $10 \mathrm{~nm}$. The transducer was oriented either on-axis or perpendicular to the trabecular meshwork for translimbal light delivery for a total of four datasets. Preliminary analysis confirmed ultrasound-determined anatomy. PA spectra were similar in all views, and data acquisition at every degree was unnecessary. Adjustments were thus made to permit acquisition of more wavelengths and compile more thorough spectroscopic information. Specifically, US/PA images were acquired every $45 \mathrm{deg}$ at wavelengths from 680 to $970 \mathrm{~nm}$ in 5-nm increments. US/PA images were postprocessed in MATLAB to determine the PA spectrum at anatomical landmarks.

\subsection{Calculating and Analyzing Measured PA Spectra}

The average PA spectra for the anterior and posterior iris, trabecular meshwork, and sclera were determined to establish PA spectral properties specific to anatomy of the anterior segment. Each spectrum was determined by calculating the average PA signal over each region of interest. Each region of interest was isolated using spatial masks determined by manual segmentation based on anatomical information depicted in US/PA images. Variations in mask size and shape had minimal impact on PA spectra; specifically, the magnitude of the variation in the PA spectra between masks was comparable to the variations in the PA spectra between eyes and was thus not considered significant. The spectrum for each region of interest was then lowpass filtered using a 10-point moving average. The resulting spectra are henceforth referred to as "measured PA spectra." Measured PA spectra were compared to established optical absorption spectra for deoxygenated hemoglobin ( $\mathrm{Hb})$, oxygenated hemoglobin $\left(\mathrm{HbO}_{2}\right)$, and melanin. ${ }^{43-49}$

\subsection{PA Analysis in Phantom Experiments of Pigment and Scattering}

The measured PA spectrum for the posterior iris required further analysis to evaluate effects of optical scattering. Following methods described elsewhere, melanin was isolated from dissected irises of fresh brown porcine eyes by collecting tissue in $10 \mathrm{~mL}$ of water and mechanically disrupting to release pigment. ${ }^{50,51}$ Pigment samples were size separated via centrifugation and isolated particles were resuspended in $2 \mathrm{~mL}$ of PBS for US/PA imaging.

A $10 \times 10 \mathrm{~cm}$ plastic container held isolated pigment samples for US/PA imaging. Two small holes were drilled on opposite sides of the container and a silicone tube (Helix Medical) with an inner diameter of 0.062 in. and an outer diameter of 0.095 in. was fed through each hole and secured. The thickness of the iris was $\sim 1 \mathrm{~mm}$ based on anatomical ultrasound images, slightly smaller than the inner diameter of the silicone tube. The tube was loaded with the following samples for US/PA imaging: PBS, iris pigment alone, and the same iris pigment with a piece of sclera wrapped around the tube. In the PA images of the circular cross-section of the tube, three regions of interest (top, middle, and bottom of the tube) were identified. The average PA signal was determined for each region of interest to assess changes in the PA spectrum of pigment due to light attenuation as a function of depth.

\subsection{Analysis of Local Tissue Composition Using Spectroscopic PA Processing}

The PA signal at each pixel, i.e., pressure $P(x, y, \lambda)$, is expressed as described elsewhere: ${ }^{25,52-55}$ 


$$
P(x, y, \lambda)=\Gamma F(x, y, \lambda) \sum_{i=1}^{n} \mu_{a_{i}}(x, y, \lambda),
$$

where $\lambda$ is the wavelength, $\mu_{a}(\lambda)$ is the optical absorption coefficient, $F(x, y, \lambda)$ is the fluence, and $\Gamma$ is the Grüneisen parameter. For heterogeneous tissue, the total PA signal at each pixel is a superposition of each PA signal from $n$ optical absorbers.

Spectroscopic PA datasets were preprocessed to correct for wavelength-dependent fluence attenuation in the iris. Fluence, $F(z, \lambda)$, is expressed as ${ }^{37,56}$

$F(z, \lambda)=F_{o}(\lambda) e^{-\mu_{\mathrm{eff}}(\lambda) \cdot z}$,

where $z$ is the imaging depth, and $F_{0}$ is the fluence at the iris surface. Effective attenuation, $\mu_{\mathrm{eff}}(\lambda)=$ $\sqrt{3 \mu_{a}(\lambda)\left[\mu_{a}(\lambda)+\mu_{s}(\lambda)(1-g(\lambda)]\right.}$, was calculated based on: (i) the coefficient of anisotropy, $g(\lambda)=2.25 \cdot \lambda^{-0.155},{ }^{57}$ (ii) the optical scattering coefficient, $\mu_{s}(\lambda)$, of $10 \%$ intralipid, ${ }^{58}$ and (iii) $\mu_{a}(\lambda)$ of melanin at the retinal pigmented epithelium (RPE). ${ }^{44,45}$

Unfortunately, published iris-specific absorption and scattering values were not available for our wavelength range. The best alternatives to estimate $\mu_{\text {eff }}$ at the iris were to use generic scattering (intralipid) and melanin absorption in the RPE. For brown eyes, $\mu_{a}[\lambda(\mathrm{nm})]=8 \cdot 10^{10} \lambda^{-3.48}\left(\mathrm{~cm}^{-1}\right)$, and for blue eyes, $\mu_{a}[\lambda(\mathrm{nm})]=6.5 \cdot 10^{10} \lambda^{-3.48}\left(\mathrm{~cm}^{-1}\right) .{ }^{44,45}$ The exponent of $\lambda$ from the expression for $\mu_{a}(\lambda)$ is the critical parameter to describe the melanin absorption spectrum and was taken directly from published data for the RPE. ${ }^{44}$ However, concentrations of melanin vary by orders of magnitude in ocular tissues, and thus the values of the leading coefficient in the expression $\mu_{a}(\lambda)$ were reduced to accommodate different melanin concentrations and distributions at the iris compared to the RPE. ${ }^{44}$ The leading coefficients were incrementally adjusted until artifacts at the posterior iris were minimized. However, processing was conservative and some artifacts were deliberately kept. Fluence correction is a complex technical issue and further work is required in the future. ${ }^{37,56}$ Using Eq. (2), $F(z, \lambda)$ was calculated to produce a spatial distribution map of fluences in the iris. The PA signal, $P$, in Eq. (1) was multiplied by $1 / F(z, \lambda)$ to correct for wavelength and depth-dependent fluence attenuation. ${ }^{37,56}$

After fluence correction, spectroscopic PA datasets were filtered using a $3 \times 3$ pixel $(41 \mu \mathrm{m} \times 45 \mu \mathrm{m})$ median filter to remove large noise spikes. Images were then low pass filtered using a $6 \times 9 \times 3$ pixel (depth $\times$ width $\times$ wavelength) moving average filter, approximately corresponding to $252 \mu \mathrm{m} \times$ $405 \mu \mathrm{m}$ and a wavelength range of $15 \mathrm{~nm}$. PA signals below the noise floor, defined by $\min (P)+0.025[\max (P)-\min (P)]$, were eliminated.

After filtering, spectroscopic PA datasets were analyzed using the linear least squares (LLS) regression method. LLS regression creates a spatial distribution map of each optical absorber in tissue. Concentration, $C$, of each absorber was estimated by separating contributions of each absorber to the total PA signal at each pixel. According to LLS regression methods described elsewhere, ${ }^{25,59,60}$ the optical absorption coefficient, $\mu_{a}(\lambda)$, in Eq. (1) can be expressed as the product of the concentration of the absorber, $C_{i}(x, y)$, and the optical cross-section of the absorber, $\sigma_{a}$. Equation (1) is rewritten as
$P(x, y, \lambda)=\Gamma F(x, y, \lambda) \sum_{i=1}^{n} C_{i}(x, y) \sigma_{a_{i}}(\lambda)$

The optical cross-section describes the likelihood an absorption event will occur, and $\sigma_{a_{i}}$ is a matrix whose rows and columns correspond to wavelength and the normalized absorption spectrum of each preselected optical absorber, respectively. For our studies, two matrices of optical cross-sections were determined a priori for LLS regression. The first matrix of optical cross-sections was obtained from the normalized absorption spectra of melanin, $\mathrm{HbO}_{2}$, and $\mathrm{Hb}$, which are reported in the literature and represent optical properties of generic tissue. ${ }^{43-49}$ The second matrix of optical cross-sections was obtained from our normalized measured PA spectra, which were specific to anterior ocular tissues. Thus, the same images were processed with LLS regression to produce two sets of concentration maps based on absorption spectra from the literature or based on our measured PA spectra.

Other research has established that it is theoretically ideal to use the maximum number of wavelengths for LLS regression of spectroscopic PA datasets. ${ }^{61,62}$ In practice, such an approach may introduce inaccuracies due to laser energy stability, noise, and overlap of optical spectra. ${ }^{61,62}$ Thus, a subset of acquired wavelengths, $\lambda=720$ to $970 \mathrm{~nm}$, was used for LLS regression of spectroscopic PA datasets. This range captured a majority of wavelengths acquired, eliminated wavelengths with energy instability, and preserved distinct features of all spectra.

\subsection{Stem Cell Tracking}

As an example application, we detected human adipose-derived mesenchymal stem cells (MSCs, Lonza, Basel, Switzerland) in the anterior eye. The measured PA spectra established here were used to identify an appropriate contrast agent to label stem cells and detect them using molecular PA imaging. Based on the PA spectra of anterior ocular tissues, established herein, gold nanospheres (AuNSs) were a good contrast agent. AuNSs with a $20 \mathrm{~nm}$ diameter were synthesized in-house as described previously, ${ }^{63}$ sterilized under UV light for at least $12 \mathrm{~h}$, and used to label MSCs following previous methods. ${ }^{63-65}$ Briefly, MSCs were maintained in $\alpha$-minimum essential medium containing $20 \%$ fetal bovine serum, penicillin, streptomycin, and L-glutamine. When MSCs reached $\sim 80 \%$ confluence, the media in the tissue culture flask was exchanged for AuNS-containing media. After $24 \mathrm{~h}$ of incubation, the AuNS-containing media was aspirated. MSCs were washed with PBS to remove residual AuNSs. The remaining AuNS-labeled MSCs were collected and suspended in PBS at a concentration of 1000 cells $/ \mu \mathrm{L}$ for a $250-\mu L$ injection.

AuNS-labeled MSCs were injected through the cornea into the anterior chamber of a porcine eye ex vivo. The control eye received no injection. To mimic in vivo conditions using an organ perfusion system, all eyes were hydrostatically clamped to maintain physiological intraocular pressures, thereby inducing circulation throughout the anterior chamber due to the natural outflow of fluid through the trabecular meshwork. US/PA datasets were acquired before and $5 \mathrm{~h}$ after injection. LLS regression of spectroscopic photoacoustic (sPA) datasets from $\lambda=680$ to $710 \mathrm{~nm}$ was performed as described in Sec. 2.5. In this case, the matrix of optical cross-sections, described in Sec. 2.5, was modified to contain the spectrum of melanin and AuNS-labeled MSCs. The spectrum of AuNS-labeled 
MSCs was determined a priori in phantom experiments according to previous methods. ${ }^{66}$

\section{Results}

Brown porcine eyes were imaged ex vivo to evaluate PA response from constituent tissues. Ultrasound images resolved anatomical features in the anterior segment, such as the sclera, cornea, lens, and iris [Figs. 1(a) and 1(b)]. Histology aided proper identification of structures [Fig. 1(c)]. Heavy pigmentation was also noted at the posterior iris [Fig. 1(c)]. By ultrasound, the cornea appeared slightly darker compared to other tissues, consistent with its relative transparency, and the interface between the cornea and sclera was distinct, reflecting differences in composition and microstructural organization of the cornea relative to other tissues. As expected, other tissues, such as the trabecular meshwork (TM), could not be unequivocally identified due to similar scattering and ultrasound speckle patterns, confirming low ultrasound contrast between most tissue types.

PA images were acquired at $700 \mathrm{~nm}$ wavelength and consecutive 45 deg cross-sections. PA signals were observed in melanin-rich tissues, namely the anterior and posterior iris and TM [Figs. 1(d)-1(g)]. Although the ciliary pigmented epithelium (CPE) is melanin-rich, no PA signal was present in this tissue due to strong attenuation of light by the overlying iris and sclera, resulting in low fluence at the CPE. PA signal was observed in some views in the scleral region, possibly corresponding to the suprachoroidal space (SCS) [Figs. 1(e) and $1(\mathrm{~g})]$. Although the sclera and SCS are not pigmented, the PA signal at these locations may result from subsurface fluence.

Spectroscopic PA datasets were acquired in brown eyes at the anterior iris and posterior iris, scleral region, and TM from $\lambda=$ 680 to $970 \mathrm{~nm}$. Representative PA spectra were plotted for fixed brown porcine eyes and distinct PA spectra were observed for each region [Fig. 2(a)]. To assess impact of fixation on the PA spectra, average PA spectra and standard deviations were calculated for multiple fresh brown porcine eyes [Figs. 2(b) and 2(c)]. PA spectra were similar between fresh and fixed eyes. The measured PA spectrum at the anterior iris closely followed the absorption spectrum of melanin reported in the literature [Fig. 2(b)]. Unexpectedly, the measured PA spectrum at the posterior iris did not follow the spectrum of melanin [Fig. 2(b)] and
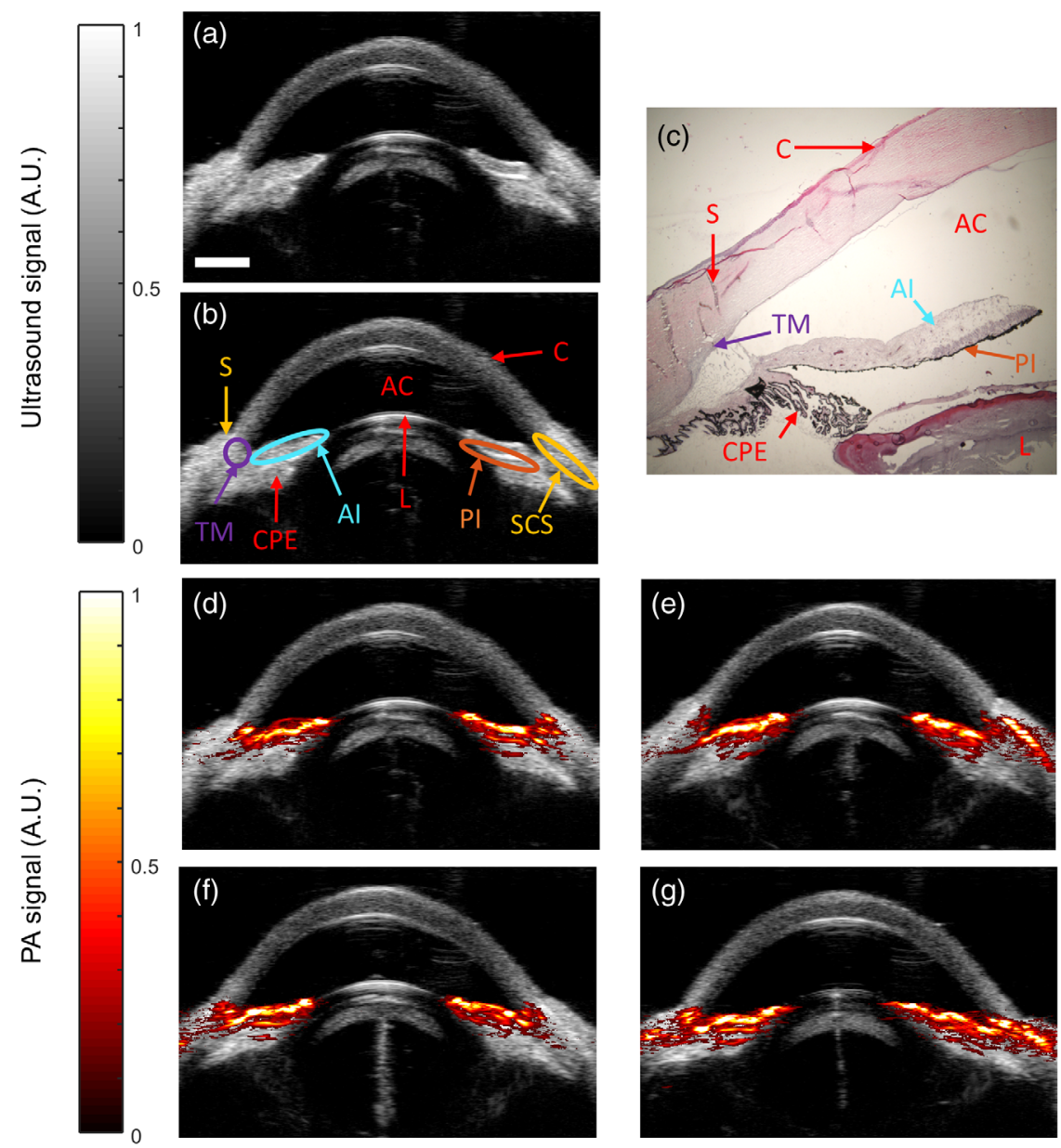

Fig. 1 Visualization of anatomy and PA signals in the anterior segment. (a, b) Grayscale ultrasound images showing anatomy of the ex vivo porcine eye. S, sclera; AC, anterior chamber; C, cornea; L, lens; TM, trabecular meshwork; AI, anterior iris; PI, posterior iris; SCS, suprachoroidal space; CPE, ciliary pigmented epithelium. (c) Corresponding histologic image of the porcine anterior segment stained with hematoxylin and eosin. (d-g) Overlay of ultrasound (gray) and PA (red) images at different locations (scans at $0 \mathrm{deg}, 45 \mathrm{deg}, 90 \mathrm{deg}$, and $135 \mathrm{deg}$ ) at $\lambda=700 \mathrm{~nm}$. PA signals appeared at melanin-rich regions, such as the TM and throughout the iris. PA signals consistently appeared at the same anatomical landmark in all frames. Scale bar for ultrasound images $=3 \mathrm{~mm}$. 
(a)

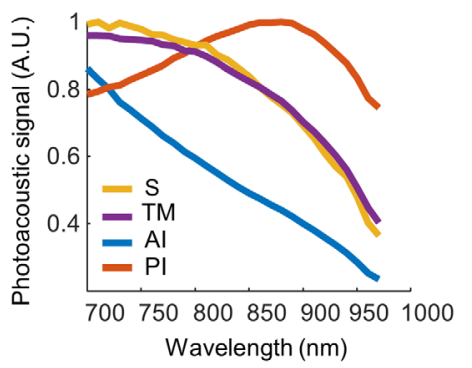

(b)

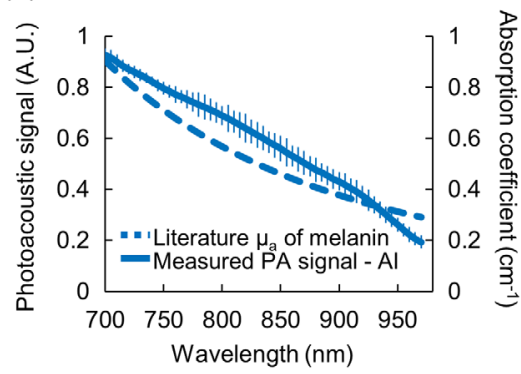

(c)

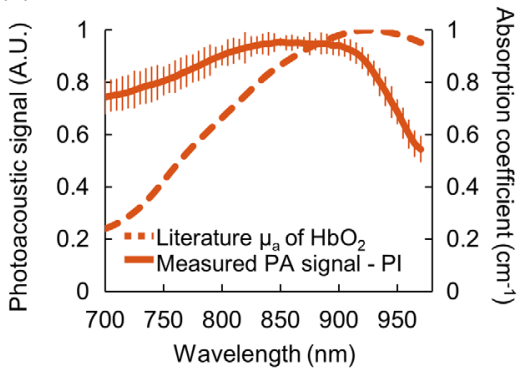

Fig. 2 PA spectra in fixed and fresh brown porcine eyes. (a) Representative PA spectra for the scleral region (S), TM, anterior iris (AI), and posterior iris (PI) for fixed brown porcine eyes. Average PA spectra and standard deviations were also calculated for (b) the anterior iris and (c) posterior iris in multiple fresh brown porcine eyes. PA spectra appeared similar in fixed and fresh eyes. The measured PA spectrum (solid blue line) of the anterior iris was compared to the reported absorption spectrum ${ }^{44,45}$ of melanin (dashed blue line). (c) The measured PA spectrum (solid orange line) of the posterior iris was compared to the reported absorption spectrum ${ }^{46-49}$ (dashed orange line) of $\mathrm{HbO}_{2}$ to highlight a potential source of PA imaging artifacts following LLS regression analysis of spectroscopic PA datasets.

showed more similarities to the literature absorption spectrum of oxygenated hemoglobin $\left(\mathrm{HbO}_{2}\right)$. Although $\mathrm{HbO}_{2}$ was not expected in ex vivo tissue, comparison with the measured PA spectrum at the posterior iris was important. Similarities between the absorption spectrum of $\mathrm{HbO}_{2}$ and the PA spectrum at the posterior iris [Fig. 2(c)] highlight a potential source of PA imaging artifacts and in turn impact use of these PA spectra to identify endogenous absorbers, select contrast agents, and facilitate molecular PA imaging.

We suspected the measured PA spectrum at the posterior iris actually represented a PA signal from melanin modified by wavelength-dependent fluence reaching the posterior iris. Therefore, fluence attenuation due to tissue absorption and scattering was studied in a silicone tube phantom. Cross-sectional US/PA images of the tube filled with purified pigment were acquired and divided into three regions of interest to study fluence attenuation and the effect on the PA spectrum of melanin at increasing depths [Fig. 3(a)]. At the top of the tube, the PA spectrum matched the literature absorption spectrum of melanin. As light was attenuated by overlying pigment, the spectrum of melanin shifted and a peak appeared at $850 \mathrm{~nm}$ at the bottom of the tube [Fig. 3(a)]. Additional scattering from scleral tissue further modified the PA spectrum of melanin [Fig. 3(b)]. Specifically, compared to attenuation primarily by optical absorption [Fig. 3(a)], the spectrum shifted at shallower depths, and a peak was observed in the middle of the tube [Fig. 3(b)]. The spectrum in the bottom region of interest further shifted with a peak closer to $900 \mathrm{~nm}$. We suspected similar fluence attenuation occurred for light propagating from the anterior to posterior iris in situ. In other words, even though the measured PA spectrum at the posterior iris should match the literature absorption spectrum of melanin, the observed PA spectrum at the posterior iris [Fig. 2(c)], resulted from wavelength-dependent light-tissue interactions.

The literature absorption spectra of melanin, $\mathrm{HbO}_{2}$, and $\mathrm{Hb}$ [Fig. 4(a)] were compared to the measured PA spectra [Fig. 4(e)] by processing spectroscopic PA datasets from brown eyes using the LLS regression method. The set of literature absorption spectra and measured PA spectra were used to obtain two different matrices of optical cross-sections. A spectrum for $\mathrm{Hb}$ could not be measured in brown eyes due to high melanin content; thus, the absorption spectrum of $\mathrm{Hb}$ from the literature was
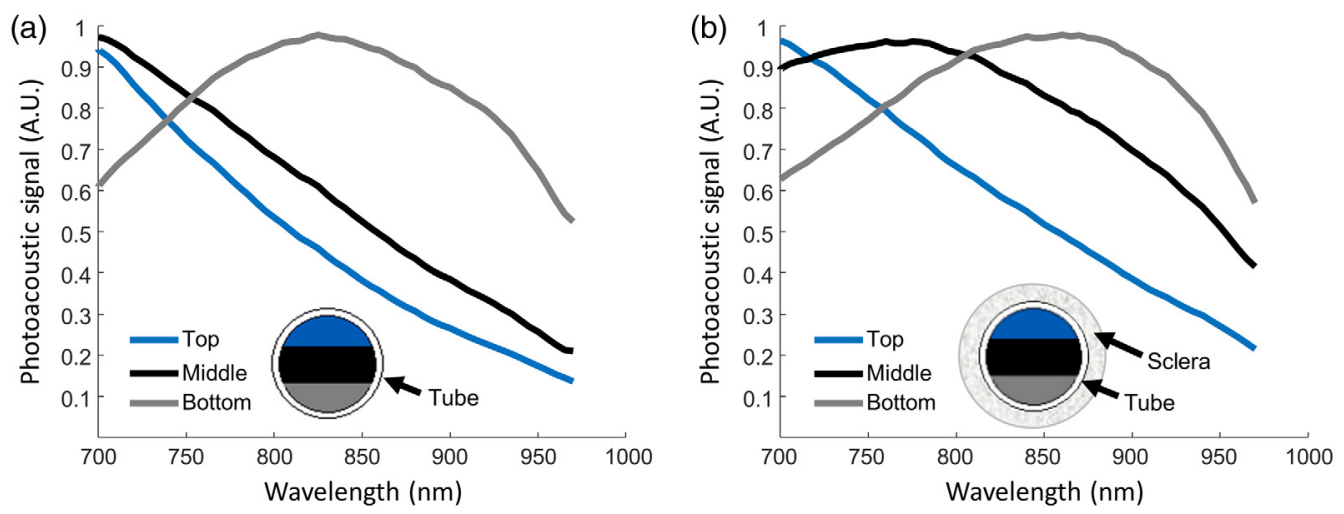

Fig. 3 PA spectrum of fresh pigment as a function of depth. A silicone tube was filled with purified pigment and cross-sectional US/PA images were acquired. The PA spectrum was analyzed within different regions of interest. "Top" and "bottom" indicate locations closest to, and further from, the transducer with the least and most light attenuation, respectively. (a) The PA spectrum of melanin was modified by depth due to light absorption of melanin, causing a significant change in the PA spectrum. (b) The experiment was repeated with the addition of a scattering layer (sclera), resulting in further modification of the measured PA spectrum of melanin. 
(a)

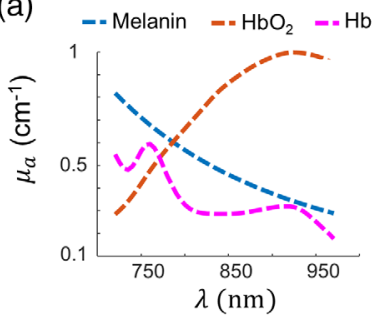

Literature
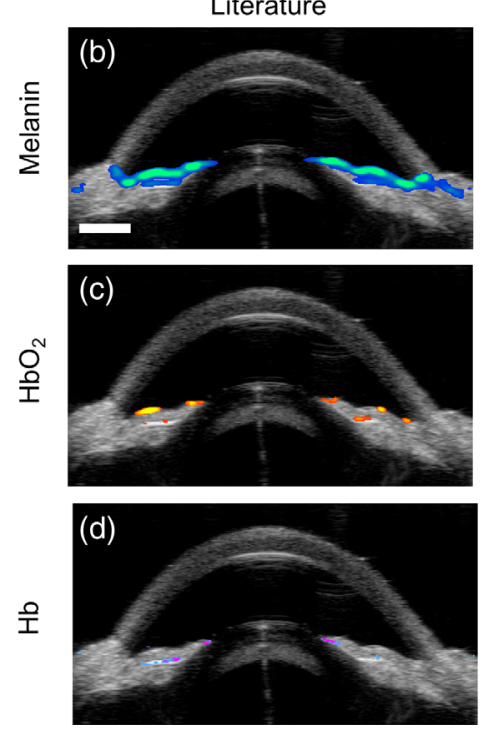

(e)

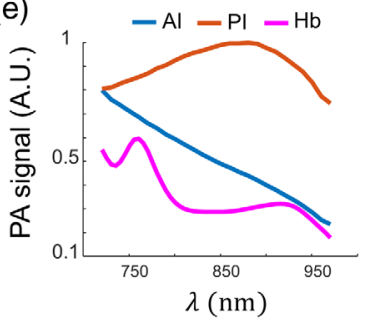

Measured
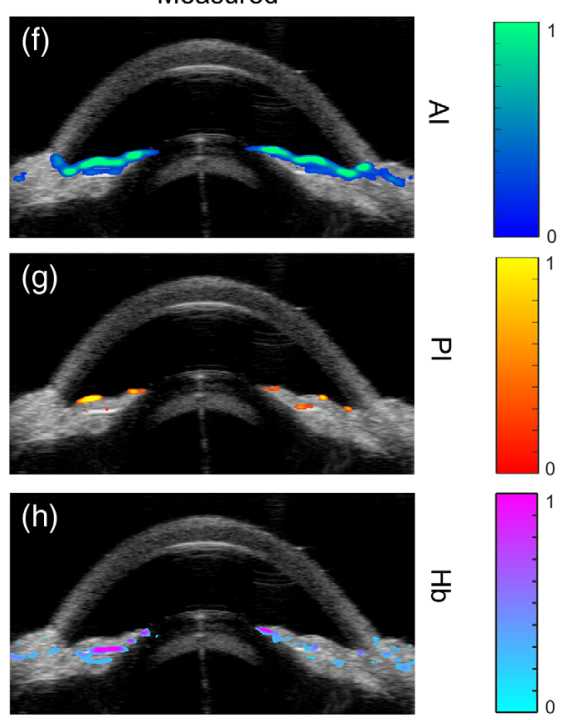

Fig. 4 sPA analysis using LLS regression in brown porcine eyes. (a) Absorption spectra from literature used for sPA analysis, ${ }^{44-49}$ and resulting computed distributions of (b) melanin, (c) $\mathrm{HbO}_{2}$, and (d) $\mathrm{Hb}$. $(\mathrm{e}-\mathrm{h})$ as in $(\mathrm{a}-\mathrm{d})$, but using measured PA spectra. Overlay of ultrasound (grayscale) and spectroscopic $\mathrm{PA}$ images showed localization of melanin (blue color scale), $\mathrm{HbO}_{2}$ (orange color scale), and $\mathrm{Hb}$ (pink color scale). Melanin was localized as expected. Absorption spectra from the literature or measured by $\mathrm{PA}$ imaging produced similar results. Some artifacts remained in the $\mathrm{HbO}_{2}$ and $\mathrm{PI}$ images (c and $\mathrm{g}$ ). Scale bar $=3 \mathrm{~mm}$.

used instead [Fig. 4(e)]. The goal of this study was to compare each set of concentration maps, generated using the literature absorption spectra, serving as the "ground truth," or our experimentally obtained measured PA spectra.

Using either set of spectra, melanin was localized as expected at the anterior iris, posterior iris, and TM [Figs. 4(b) and 4(f)]. Because $\mathrm{HbO}_{2}$ was not expected in post-mortem tissue, pixels indicating presence of $\mathrm{HbO}_{2}$ were determined to be image artifacts [Fig. 4(c)]. Agreement between the concentration maps for $\mathrm{HbO}_{2}$ [Fig. 4(c)] and the posterior iris [Fig. 4(g)] indicated the artifact occurred due to the measured spectrum at the posterior iris being most similar to that of $\mathrm{HbO}_{2}$ among the species we considered (melanin, $\mathrm{Hb}$, and $\mathrm{HbO}_{2}$ ); however, the match is only very approximate [Fig. 2(c)]. Although the posterior iris is a melanin-rich tissue, the PA spectrum was modified by fluence attenuation. Without fluence correction, LLS regression predicted a prominent $\mathrm{HbO}_{2}$ distribution at the posterior iris (data not shown). By implementing fluence correction, melanin was accurately localized to the anterior iris [Figs. 4(b) and 4(f)] and the presence of $\mathrm{HbO}_{2}$ was minimized at the posterior iris [Figs. 4(c) and 4(g)]. Hb was primarily localized to the posterior iris, but some discrepancies were observed between distribution maps [Figs. 4(d) and 4(h)]. LLS regression of sPA datasets from multiple brown eyes produced similar results, indicating PA properties were similar across various colorations of brown eyes from light to dark. Agreement between both sets of concentration maps [Figs. 4(b)-4(d) and 4(f)-4(h)] impacts future use of measured PA spectra for molecular PA imaging. Similar concentration maps across multiple brown eyes indicated our measured PA spectra can be used to inform contrast agent selection for a particular application according to endogenous absorber distribution.

Characterization and analysis of PA properties was repeated in blue porcine eyes (Fig. 5). Each spectrum in brown and blue eyes was normalized according to its peak absorption to better visualize the PA properties in different eye colors. Results do not depict differences in magnitude of PA signals. The posterior iris was the only structure to consistently show a PA signal in the blue eye at the 700-nm wavelength [Fig. 5(a)]. Due to less melanin than in brown eyes, PA signals were not evident in other tissues. The PA signal from melanin at the posterior iris was modified by fluence attenuation, and thus a similar artifact was observed in the blue eye [Fig. 5(b)] as in brown eyes. Other types of PA spectra were also occasionally observed in blue eyes [Figs. 5(c) and 5(d)] but were not localized to a consistent location, i.e., the spectrum shown in Fig. 5(b) was most prevalent in blue eyes.

Spectroscopic PA analysis using the LLS regression method was repeated for blue eyes (Fig. 6). In the case of blue eyes, sPA analysis was conducted using the measured PA spectra from brown eye datasets, except for the spectrum of $\mathrm{Hb}$, which was possible to isolate in a blue eye. Spectroscopic PA datasets 
(a)
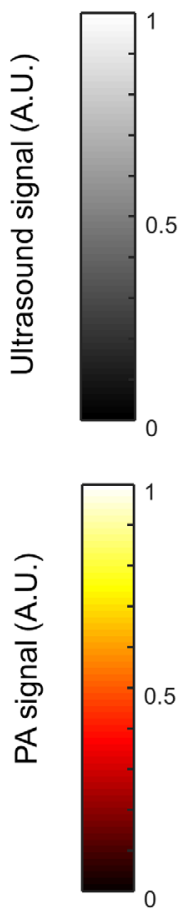

(c)
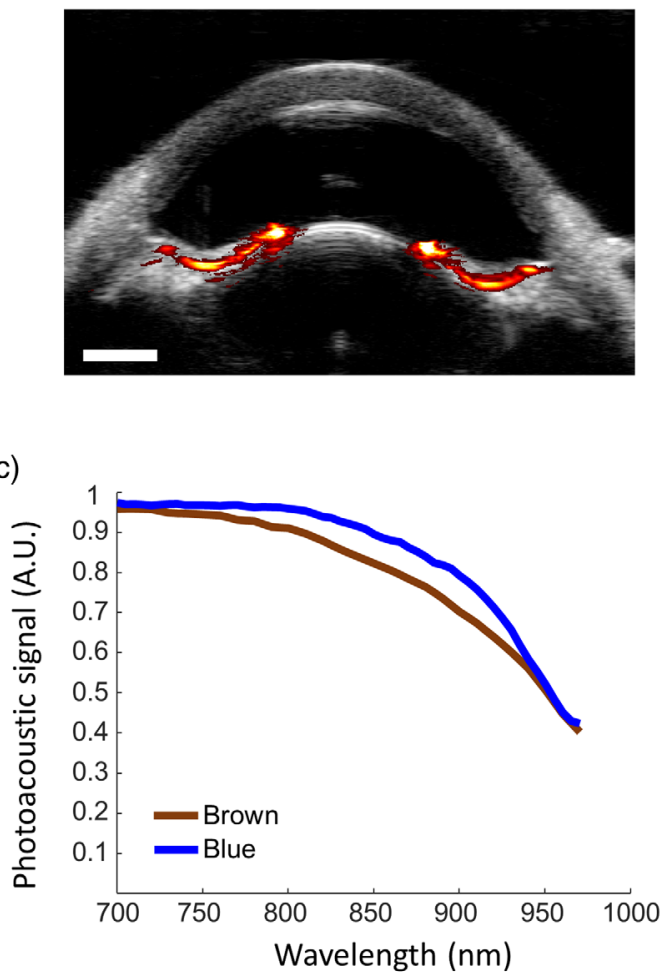

(b)

(d)
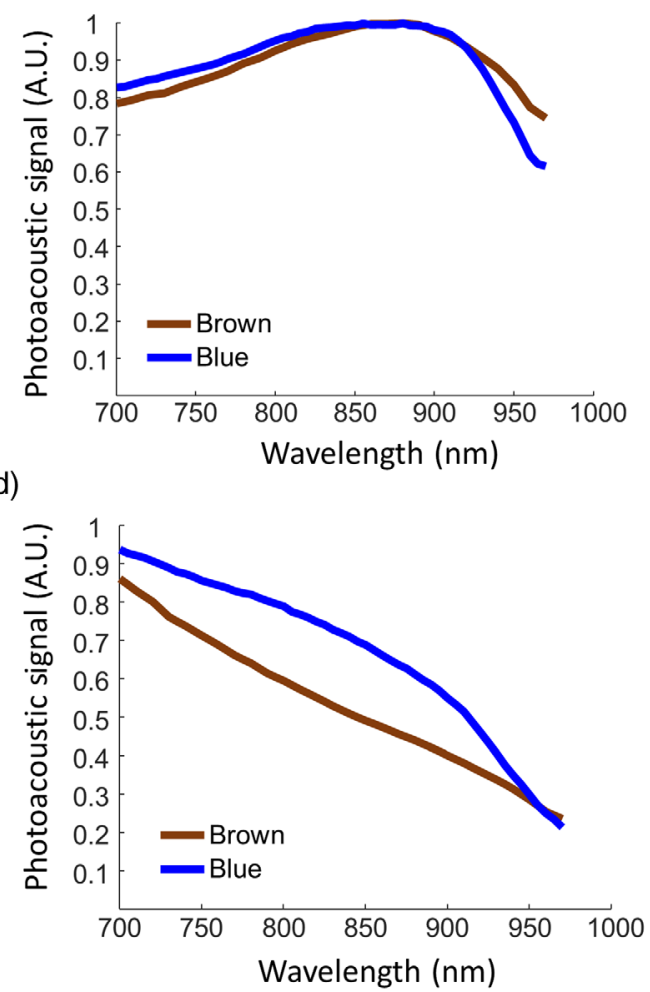

Fig. 5 Comparison of measured PA spectra in blue and brown eyes. (a) Overlay of ultrasound (gray) and phototacoustic (red) images in a blue porcine eye. PA signal was localized to the posterior iris. (b-d) Spectra were separated based on results from brown porcine eyes. (b) A modified melanin spectrum, resembling that of oxygenated hemoglobin, was isolated to the posterior iris in both eye colors and was the dominant spectrum observed in blue eyes. (c and d) Other types of spectra were observed in blue eyes, but were not localized to a consistent location. (d) The PA spectrum resembling melanin (b) was most prevalent in brown eyes. Scale bar $=3 \mathrm{~mm}$.

from blue porcine eyes were similarly processed using either the literature absorption spectra [Fig. 6(a)] or the measured PA spectra [Fig. 6(e)] to identify absorbers. After correcting for wavelength-dependent fluence attenuation, melanin was accurately localized at the iris [Figs. 6(b) and 6(f)]. Some effects of fluence attenuation remained [Figs. 6(c) and 6(g)]. However, fluence correction increased melanin localization at the posterior iris [Figs. 6(b) and 6(f)] and minimized the presence of $\mathrm{HbO}_{2}$ [Fig. 6(c)] and the modified melanin spectrum at the posterior iris [Fig. 6(g)]. Hb was also identified [Figs. 6(d) and 6(h)]. sPA analysis in blue eyes accurately localized absorbers. In this case, agreement between concentration maps [Figs. 6(b)$6(\mathrm{~d})$ and $6(\mathrm{f})-6(\mathrm{~h})$ ] indicated conservation of PA properties across drastically different eye colors.

The PA properties of anterior segment tissues reported here can be used for a variety of applications to facilitate molecular PA imaging in the anterior eye. One such application is PA tracking of injected stem cells to regenerate the trabecular meshwork in glaucoma patients. ${ }^{40}$ In a preliminary study, MSCs were labeled with AuNSs, injected into the anterior chamber of a porcine eye, and allowed to circulate for $5 \mathrm{~h}$. A separate eye, which was not injected with AuNS-labeled MSCs but otherwise followed the same experimental protocol, was used as a control. US/PA imaging and SPA analysis using LLS regression was conducted to separate signals from melanin (the primary endogenous absorber of interest in this case) and injected stem cells (Fig. 7). Prior to injection, melanin was accurately localized throughout the anterior segment [Fig. 7(a)] and no AuNSlabeled MSCs were detected [Fig. 7(b)]. This validates accuracy of the algorithm and confirms minimal overlap of the spectrum of the contrast agent with the spectrum of melanin. At $5 \mathrm{~h}$ postinjection, sPA analysis accurately localized melanin at the iris [Fig. 7(c)]. AuNS-labeled MSCs were detected lining the lens [Fig. 7(d)]. The measured PA spectra described here directly informed contrast agent selection to allow successful stem cell detection in an organ perfusion system that mimics in vivo conditions. Therefore, ex vivo analysis was applicable to a more clinically relevant organ perfusion system, and the measured PA spectra will continue to be used to improve upon this proof-of-concept stem cell tracking platform.

\section{Discussion}

Clinically available ophthalmic imaging modalities such as OCT and ultrasound provide excellent anatomical information for ocular tissues. ${ }^{9,10,67}$ However, development of molecular imaging techniques has not developed as rapidly, particularly for the anterior segment. ${ }^{16,17} \mathrm{PA}$ imaging is one option to address the need for molecular imaging in the anterior eye. Implementation of molecular PA imaging requires use of endogenous or exogenous contrast agents. ${ }^{26,27}$ Contrast agents must be carefully selected based on the application of interest and PA properties of the surrounding tissues. Without prior knowledge of PA properties of anterior segment tissues, it is impossible to ensure a contrast agent can be distinguished from background 

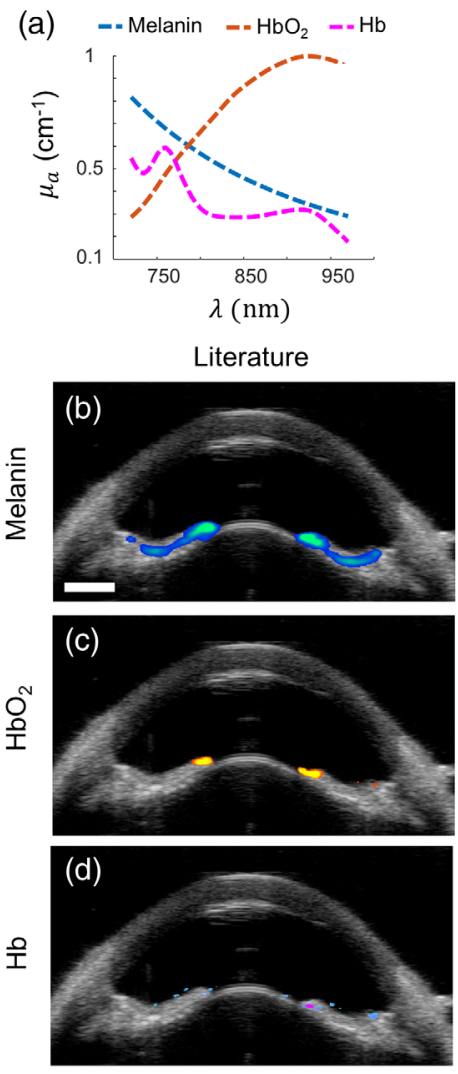
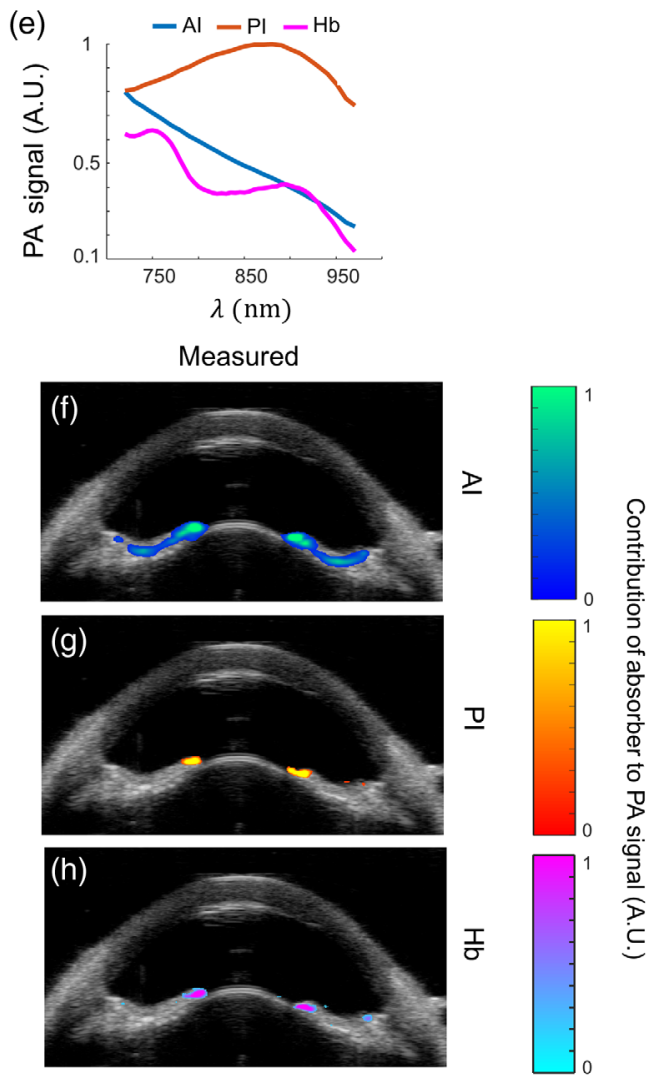

Fig. 6 Spectroscopic PA (sPA) analysis using LLS regression in blue porcine eyes. (a) Literature absorption spectra used for SPA analysis. ${ }^{4-49}$ (b-d) SPA analysis using the literature absorption spectra. (e) Measured PA spectra used for sPA analysis, which were compiled from brown eye datasets, except for the spectrum of $\mathrm{Hb}$. ( $\mathrm{f}-\mathrm{h}) \mathrm{SPA}$ analysis using the measured PA spectra. Overlay of ultrasound (grayscale) and spectroscopic PA images showed localization of melanin (blue colorscale), $\mathrm{HbO}_{2}$ (orange colorscale), and $\mathrm{Hb}$ (pink colorscale). Absorbers were localized as expected according to known anatomy. Literature absorption spectra or measured PA spectra produced similar results, and either can be applied for SPA analysis. These results show that the PA spectra can be used for SPA analysis in both brown and blue eyes. Scale bar $=3 \mathrm{~mm}$.
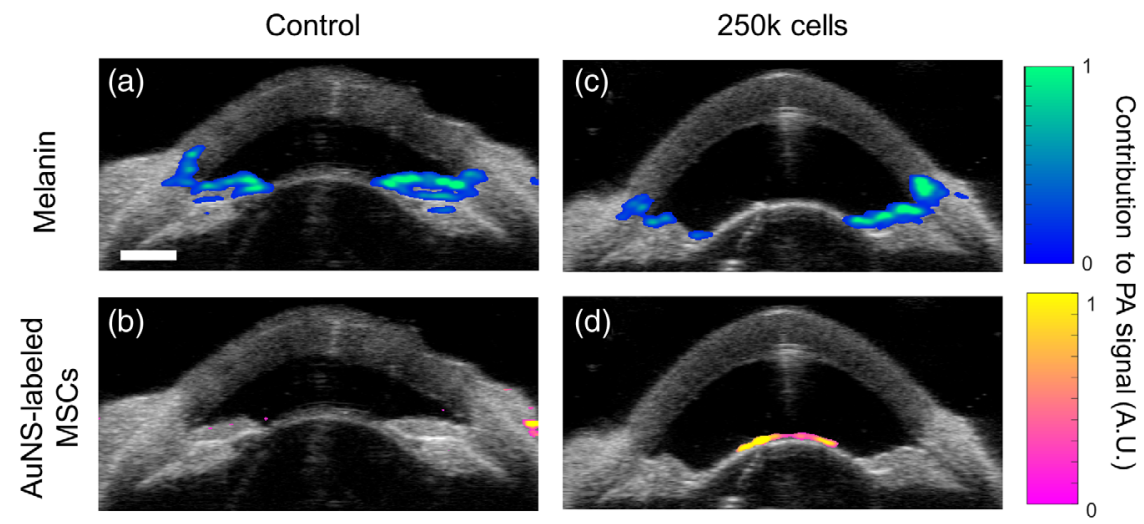

Fig. 7 Proof-of-concept demonstration of stem cell tracking in the anterior eye. Stem cells must be labeled with a contrast agent in culture, prior to injection, to allow detection with PA imaging. The measured PA spectra established here and concentration maps of endogenous absorbers were used to inform contrast agent selection. Accordingly, stem cells were labeled with AuNSs. To mimic in vivo conditions, enucleated brown porcine eyes were perfused to maintain physiological pressure and were perfused to maintain physiological pressure and were imaged before $(a, b)$ and $5 \mathrm{~h}$ after $(c, d)$ injection of AuNS-labeled stem cells. SPA analysis using LLS regression separated PA signals from $(a, c)$ melanin and (b, d) AuNS-labeled stem cells. (b) Minor artifacts were observed at the sclera, but the location is outside of the anterior chamber and AuNS-labeled MSCs would not be present in this region. Scale bar $=3 \mathrm{~mm}$. 
PA signals. Thus, the first step to extend molecular PA imaging to the anterior eye is to establish PA spectral properties.

We here systematically analyzed PA properties of anterior segment tissues to produce a set of measured PA spectra in the first optical window between 680 and $970 \mathrm{~nm}$ to facilitate molecular PA imaging. Distinct PA spectra were observed at the $\mathrm{TM}$, anterior iris, posterior iris, and scleral region in brown porcine eyes. Similar PA spectra were observed at the posterior iris in blue porcine eyes. These measured PA spectra were compared to literature absorption spectra by analyzing sPA datasets of blue and brown porcine eyes using LLS regression to produce concentration maps of each absorber. Good agreement was observed between absorber maps, which validates future use of our PA spectra to facilitate molecular PA imaging in vivo.

We hypothesized that each measured PA spectrum would match a known absorption spectrum of an endogenous absorber, specifically melanin. However, this was only true for the anterior iris in brown eyes. In other tissues, the PA spectrum was modified by wavelength- and depth-dependent fluence attenuation. These effects were most prominent at the posterior iris, especially in blue eyes. The impact of fluence attenuation on the PA spectrum at the iris was consistent with results from a simplified phantom experiment. Without fluence correction, sPA analysis using LLS regression contained many artifacts and mistakenly attributed PA signals to light absorption by $\mathrm{HbO}_{2}$, which coincidentally has a spectrum similar to the fluence-modified melanin spectrum. Wavelength- and depth-dependent fluence correction allowed better PA identification of melanin in the anterior segment and minimized artifacts. Further improvement to the fluence correction method is possible. Specifically, we assumed a uniform concentration of melanin, but concentration varies spatially throughout the iris. In addition, at the wavelengths of interest (680 to $970 \mathrm{~nm}$ ), information on melanin absorption and scattering at the iris were unknown. Thus, optical properties of the iris were estimated based on available data for the RPE and a general model of scattering (intralipid) as substitutes. ${ }^{43-45,57,58}$ This motivates further study so that parameters specific to the iris can be determined. We anticipate the effects of fluence attenuation observed here will impact molecular PA imaging strategies in the future.

Comparison between blue and brown porcine eyes indicated that the set of measured PA spectra were independent of eye color, at least in the near-infrared region. This result was fortuitous but not obvious a priori. Although blue and brown eyes have the same type of pigment, in the visible spectrum differences in melanin concentration and scattering produce variations in eye color. In spite of distinct optical properties in the visible regime, PA properties in the near-infrared regime were similar across eye colors. This result was important to confirm our measured PA spectra were applicable to both extremes in eye color (blue and brown). Thus, a single set of measured PA spectra can possibly inform molecular PA imaging strategies across the full spectrum of eye colors. Each eye color does not appear to require a unique set of spectra to predict endogenous absorber distribution and background PA signals to inform contrast agent selection. If this were the case, implementing molecular PA imaging in the anterior eye would likely be impractical.

Finally, we demonstrated how the measured PA spectra can be used for one application of molecular PA imaging, tracking stem cells in the anterior segment for glaucoma treatment. ${ }^{40-42}$ In this application, stem cells were labeled with a contrast agent in culture, before injection, to demonstrate molecular PA imaging. ${ }^{63}$ Thus, prior knowledge of endogenous absorber distribution and background PA signals was required to ensure stem cells were labeled with an appropriate contrast agent for accurate detection. ${ }^{26,27}$ Fortunately, in this particular application, wavelength-dependent fluence attenuation observed at the posterior iris was irrelevant because stem cells will be localized more anteriorly. Based on our measured PA spectra and endogenous absorber concentration maps, AuNSs were a good option for the contrast agent. Results of this proof-of-concept study indicated stem cells labeled with AuNSs were successfully distinguished from background PA signals. Furthermore, the stem cell imaging study was conducted in a clinically relevant model system: a perfused porcine eye under physiological pressure, which closely mimics in vivo conditions. Use of organ culture systems is well established in ophthalmic research due to similarities between porcine, monkey, and human ocular anatomy, and organ culture systems provide a valuable intermediary step toward the development of in vivo imaging techniques. ${ }^{68-70}$ The fact that the measured PA spectra established here allowed detection of stem cells in a perfusion setup further supports the impact of our results on future development of molecular PA imaging techniques for the anterior eye.

Although AuNSs were a good choice for stem cell tracking, other applications may have different requirements for molecular PA imaging. For example, if a researcher is interested in studying iridial pathologies, contrast agent options will vary according to the unique background PA signals at the iris. Beyond contrast agent selection and molecular PA imaging, the PA spectra could also serve as a baseline to evaluate spectral changes related to tissue health. In the future, we will use the measured PA spectra described herein to evaluate other contrast agents for stem cell tracking, aid development of light delivery systems for the anterior eye, and investigate therapeutic use of light to develop image-guided therapy platforms.

\section{Conclusions}

PA tissue properties were analyzed throughout the anterior segment. Measured PA spectra were compiled based on distinct PA spectra observed in the anterior and posterior iris, TM, and sclera. These spectra were compared to existing published absorption spectra. sPA analysis using both the measured PA spectra and published absorption spectra accurately localized absorbers throughout the anterior segment. In addition, the measured PA spectra from brown eyes were used for sPA analysis of datasets from blue eyes. Although there are many eye colors in the visible range, our comparison of blue and brown porcine eyes indicated anterior segments are photoacoustically similar in the near-infrared wavelength range. Results have important implications for development of molecular PA imaging strategies and other light-based imaging techniques and therapies in the anterior segment, including our particular application of interest, stem cell tracking for glaucoma treatment.

\section{Disclosures}

None.

\section{Acknowledgments}

The authors acknowledge support from the Georgia Research Alliance (GRA) and the National Science Foundation (NSF). 


\section{References}

1. J. L. Ramos, Y. Li, and D. Huang, "Clinical and research applications of anterior segment optical coherence tomography: a review," Clin. Exp. Ophthalmol. 37(1), 81-89 (2009).

2. A. M. Zysk et al., "Optical coherence tomography: a review of clinical development from bench to bedside," J. Biomed. Opt. 12(5), 051403 (2007).

3. C. Bianciotto et al., "Assessment of anterior segment tumors with ultrasound biomicroscopy versus anterior segment optical coherence tomography in 200 cases," Ophthalmology 118(7), 1297-1302 (2011).

4. T. Dada et al., "Comparison of anterior segment optical coherence tomography and ultrasound biomicroscopy for assessment of the anterior segment," J. Cataract Refractive Surg. 33(5), 837-840 (2007).

5. S. Radhakrishnan et al., "Comparison of optical coherence tomography and ultrasound biomicroscopy for detection of narrow anterior chamber angles," Arch. Ophthalmol. 123(8), 1053-1059 (2005).

6. D. Huang et al., "Optical coherence tomography," Science 254(5035), 1178-1181 (1991).

7. M. R. Hee et al., "Optical coherence tomography of the human retina," Arch. Ophthalmol. 113(3), 325-332 (1995)

8. E. A. Swanson et al., "In vivo retinal imaging by optical coherence tomography," Opt. Lett. 18(21), 1864-1866 (1993).

9. C. J. Pavlin et al., "Clinical use of ultrasound biomicroscopy," Ophthalmology 98(3), 287-295 (1991).

10. A. Konstantopoulos, P. Hossain, and D. F. Anderson, "Recent advances in ophthalmic anterior segment imaging: a new era for ophthalmic diagnosis?" Br. J. Ophthalmol. 91(4), 551-557 (2007).

11. S. Radhakrishnan et al., "Real-time optical coherence tomography of the anterior segment at $1310 \mathrm{~nm}, "$ Arch. Ophthalmol. 119(8), 11791185 (2001).

12. B. Cense et al., "Thickness and birefringence of healthy retinal nerve fiber layer tissue measured with polarization-sensitive optical coherence tomography," Invest. Opthalmol. Visual Sci. 45(8), 2606-2612 (2004).

13. I. Grulkowski et al., "Retinal, anterior segment and full eye imaging using ultrahigh speed swept source OCT with vertical-cavity surface emitting lasers," Biomed. Opt. Express 3(11), 2733-2751 (2012).

14. B. Potsaid et al., "Ultrahigh speed $1050 \mathrm{~nm}$ swept source/Fourier domain OCT retinal and anterior segment imaging at 100,000 to 400,000 axial scans per second," Opt. Express 18(19), 20029-20048 (2010).

15. Y. Diebold and M. Calonge, "Applications of nanoparticles in ophthalmology," Prog. Retinal Eye Res. 29(6), 596-609 (2010).

16. S. A. Boppart et al., "Optical probes and techniques for molecular contrast enhancement in coherence imaging," J. Biomed. Opt. 10(4), 041208 (2005).

17. A. L. Oldenburg et al., "Molecular optical coherence tomography contrast enhancement and imaging," in Optical Coherence Tomography, W. Drexler and J. Fujimoto, Eds., pp. 1429-1454, Springer, Cham (2015).

18. R. K. Manapuram et al., "In vivo estimation of elastic wave parameters using phase-stabilized swept source optical coherence elastography," J. Biomed. Opt. 17(10), 100501 (2012).

19. M. Mehrmohammadi et al., "Pulsed magneto-motive ultrasound imaging using ultrasmall magnetic nanoprobes," Mol. Imaging 10(2), 102-110 (2011)

20. M. Lapierre-Landry et al., "In vivo photothermal optical coherence tomography of endogenous and exogenous contrast agents in the eye," Sci. Rep. 7(1), 9228 (2017).

21. M. Lapierre-Landry et al., "Imaging melanin distribution in the zebrafish retina using photothermal optical coherence tomography," Transl. Vision Sci. Technol. 7(5), 4 (2018).

22. M. Lapierre-Landry et al., "Photothermal optical coherence tomography of indocyanine green in ex vivo eyes," Opt. Lett. 43(11), 2470-2473 (2018).

23. M. Lapierre-Landry et al., "In vivo photothermal optical coherence tomography of gold nanorods in the mouse eye," in Opt. Life Sci. Cong., OSA, Washington, D.C., p. BoM3A.2 (2017).

24. A. G. Bell, "LXVIII. Upon the production of sound by radiant energy," London Edinburgh Dublin Philos. Mag. J. Sci. 11(71), 510-528 (1881).

25. M. Xu and L. V. Wang, "Photoacoustic imaging in biomedicine," Rev. Sci. Instrum. 77(4), 041101 (2006).

26. S. Y. Emelianov, P.-C. Li, and M. O'Donnell, "Photoacoustics for molecular imaging and therapy," Phys. Today 62(5), 34-39 (2009).
27. G. P. Luke, D. Yeager, and S. Y. Emelianov, "Biomedical applications of photoacoustic imaging with exogenous contrast agents," Ann. Biomed. Eng. 40(2), 422-437 (2012).

28. C. J. Pavlin, K. Harasiewicz, and F. S. Foster, "Ultrasound biomicroscopy of anterior segment structures in normal and glaucomatous eyes," Am. J. Ophthalmol. 113(4), 381-389 (1992).

29. S. Jiao et al., "Photoacoustic ophthalmoscopy for in vivo retinal imaging," Opt. Express 18(4), 3967-3972 (2010).

30. W. Song et al., "Integrating photoacoustic ophthalmoscopy with scanning laser ophthalmoscopy, optical coherence tomography, and fluorescein angiography for a multimodal retinal imaging platform," J. Biomed. Opt. 17(6), 061206 (2012).

31. C. L. Bayer et al., "Multiplex photoacoustic molecular imaging using targeted silica-coated gold nanorods," Biomed. Opt. Express 2(7), 1828-1835 (2011)

32. K. Homan et al., "Silver nanosystems for photoacoustic imaging and image-guided therapy," J. Biomed. Opt. 15(2), 021316 (2010).

33. J. Su et al., "Photoacoustic imaging of clinical metal needles in tissue," J. Biomed. Opt. 15(2), 021309 (2010).

34. R. H. Silverman et al., "High-resolution photoacoustic imaging of ocular tissues," Ultrasound Med. Biol. 36(5), 733-742 (2010).

35. S. Y. Nam and S. Y. Emelianov, "Array-based real-time ultrasound and photoacoustic ocular imaging," J. Opt. Soc. Korea 18(2), 151-155 (2014)

36. A. de la Zerda et al., "Photoacoustic ocular imaging," Opt. Lett. 35(3), 270-272 (2010)

37. K. Maslov, H. F. Zhang, and L. V. Wang, "Effects of wavelengthdependent fluence attenuation on the noninvasive photoacoustic imaging of hemoglobin oxygen saturation in subcutaneous vasculature in vivo," Inverse Probl. 23(6), S113-S122 (2007).

38. R. A. Sturm and M. Larsson, "Genetics of human iris colour and patterns," Pigment Cell Melanoma Res. 22(5), 544-562 (2009).

39. P. D. Imesch, I. H. Wallow, and D. M. Albert, "The color of the human eye: a review of morphologic correlates and of some conditions that affect iridial pigmentation," Surv. Ophthalmol. 41, S117-S123 (1997).

40. E. J. Snider et al., "Characterizing differences between MSCs and TM cells: toward autologous stem cell therapies for the glaucomatous trabecular meshwork," J. Tissue Eng. Regener. Med. 12(3), 695-704 (2018)

41. R. Manuguerra-Gagné et al., "Transplantation of mesenchymal stem cells promotes tissue regeneration in a glaucoma model through laser-induced paracrine factor secretion and progenitor cell recruitment," Stem Cells 31(6), 1136-1148 (2013).

42. D. W. Abu-Hassan et al., "Induced pluripotent stem cells restore function in a human cell loss model of open-angle glaucoma," Stem Cells 33(3), 751-761 (2015).

43. S. L. Jacques, "Optical properties of biological tissues: a review," Phys. Med. Biol. 58(11), R37-R61 (2013).

44. S. L. Jacques, R. D. Glickman, and J. A. Schwartz, "Internal absorption coefficient and threshold for pulsed laser disruption of melanosomes isolated from retinal pigment epithelium," Proc. SPIE 2681, 468-477 (1996).

45. S. L. Jacques and D. J. McAuliffe, "The melanosome: threshold temperature for explosive vaporization and internal absorption coefficient during pulsed laser irradiation," Photochem. Photobiol. 53(6), 769-775 (1991)

46. M. K. Moaveni, "A multiple scattering field theory applied to whole blood," PhD Dissertation, Department of Electrical Engineering, University of Washington (1970).

47. J. M. Schmitt, "Optical measurement of blood oxygenation by implantable telemetry," Technical Report G5558-15, Stanford (1986).

48. S. Takatani and M. D. Graham, "Theoretical analysis of diffuse reflectance from a two-layer tissue model," IEEE Trans. Biomed. Eng. BME26(12), 656-664 (1979).

49. S. Prahl, "Optical absorption of hemoglobin," 1999, https://omlc.org/ spectra/hemoglobin/index.html (11 July 2018).

50. K. M. Porter, D. L. Epstein, and P. B. Liton, "Up-regulated expression of extracellular matrix remodeling genes in phagocytically challenged trabecular meshwork cells," PLoS One 7(4), e34792 (2012).

51. D. L. Epstein et al., "Experimental obstruction to aqueous outflow by pigment particles in living monkeys," Invest. Ophthalmol. Visual Sci. 27(3), 387-395 (1986). 
52. T. Bowen et al., "Some experimental results on the thermoacoustic imaging of tissue equivalent phantom materials," in Ultrason. Symp., IEEE, pp. 823-827 (1981).

53. X. Wang et al., "Three-dimensional laser-induced photoacoustic tomography of mouse brain with the skin and skull intact," Opt. Lett. 28(19), 1739-1741 (2003).

54. R. A. Kruger and P. Liu, "Photoacoustic ultrasound: pulse production and detection in 0.5\% Liposyn," Med. Phys. 21(7), 1179-1184 (1994).

55. A. A. Oraevsky et al., "Laser-based optoacoustic imaging in biological tissues," Proc. SPIE 2134, 122-128 (1994).

56. B. Cox et al., "Quantitative spectroscopic photoacoustic imaging: a review," J. Biomed. Opt. 17(6), 061202 (2012).

57. S. T. Flock et al., "Optical properties of intralipid: a phantom medium for light propagation studies," Lasers Surg. Med. 12(5), 510-519 (1992).

58. H. J. van Staveren et al., "Light scattering in Intralipid-10\% in the wavelength range of 400-1100 nm," Appl. Opt. 30(31), 4507-4514 (1991).

59. X. Wang et al., "Noninvasive laser-induced photoacoustic tomography for structural and functional in vivo imaging of the brain," Nat. Biotechnol. 21(7), 803-806 (2003).

60. L. V. Wang, Photoacoustic Imaging and Spectroscopy, CRC Press, Boca Raton, Florida (2009).

61. G. P. Luke and S. Y. Emelianov, "Optimization of in vivo spectroscopic photoacoustic imaging by smart optical wavelength selection," Opt. Lett. 39(7), 2214-2217 (2014).

62. G. P. Luke, S. Y. Nam, and S. Y. Emelianov, "Optical wavelength selection for improved spectroscopic photoacoustic imaging," Photoacoustics 1(2), 36-42 (2013).

63. S. Y. Nam et al., "In vivo ultrasound and photoacoustic monitoring of mesenchymal stem cells labeled with gold nanotracers," PLoS One 7(5), e37267 (2012).

64. L. M. Ricles et al., "Function of mesenchymal stem cells following loading of gold nanotracers," Int. J. Nanomed. 6, 407-416 (2011).

65. L. M. Ricles et al., "A dual gold nanoparticle system for mesenchymal stem cell tracking," J. Mater. Chem. B 2(46), 8220-8230 (2014).

66. S. Y. Nam et al., "Nonlinear photoacoustic signal increase from endocytosis of gold nanoparticles," Opt. Lett. 37(22), 4708-4710 (2012).

67. J. S. Schuman et al., Optical Coherence Tomography of Ocular Diseases, Slack, New Jersey (2004).
68. S. K. Bhattacharya et al., "Cochlin expression in anterior segment organ culture models after TGF 32 treatment," Invest. Ophthalmol. Visual Sci. 50(2), 551-559 (2009).

69. D. H. Johnson and R. C. Tschumper, "The effect of organ culture on human trabecular meshwork," Exp. Eye Res. 49(1), 113-127 (1989).

70. D. Johnson and R. Tschumper, "Human trabecular meshwork organ culture. A new method," Invest. Ophthalmol. Visual Sci. 28(6), 945953 (1987).

Kelsey P. Kubelick is a PhD candidate in the Coulter Department of Biomedical Engineering at the Georgia Institute of Technology and Emory University. Her research focuses on development of cell and particle tracking approaches using ultrasound, photoacoustic, and magnetic resonance imaging to expedite translation of novel therapies, particularly in the fields of regenerative medicine and immunology.

Eric J. Snider received his doctorate in biomedical engineering at Georgia Institute of Technology/Emory University, where his research took steps towards developing stem cell therapies for the glaucomatous trabecular meshwork. He is currently a postdoctoral fellow at the United States Army Institute of Surgical Research, focused on developing therapeutics and diagnostics for ocular trauma.

C. Ross Ethier holds the Lawrence L. Gellerstedt, Jr. chair in bioengineering and is a Georgia Research Alliance Eminent Scholar in the Coulter Department of Biomedical Engineering at Georgia Tech/ Emory. His research is in the biomechanics of cells and whole organs, with specific emphasis on ocular biomechanics. He works on developing treatments for glaucoma, the second most common cause of blindness, and for SANS, a syndrome affecting astronauts that is a major NASA human health concern.

Stanislav Emelianov is a Joseph M. Pettit Endowed Chair, Georgia Research Alliance Eminent Scholar, and professor of electrical and computer engineering and biomedical engineering at the Georgia Institute of Technology and Emory University School of Medicine. Furthermore, he is director of the Ultrasound Imaging and Therapeutics Research Laboratory. Projects in his laboratory are focused on the discovery, development, and clinical translation of diagnostic imaging and therapeutic instrumentation, augmented with theranostic nanoagents. 